\title{
On the Eilenberg-MacLane invariants of loop spaces.
}

\author{
By Haruo SuzUKI
}

(Received Dec. 16, 1954)

1. Let $X$ be a simply connected topological space and let $E$ be the space of all paths in $X$ starting from a fixed point $x_{0} \in X$, topologized by compact-open topology. Then $E$ is contractible, and with the projection $\rho: E \rightarrow X$ which associates each path to its terminal point, $(E, \rho, X)$ is a fiber space in the sense of Serre [1], where the fiber at $x_{0}$ is the loop space $\Omega_{X}$ of $X$. It is well known that we have $\pi_{i}(X) \approx \pi_{i-1}\left(\Omega_{X}\right), i=2,3, \cdots$.

Fixing integers $p, q$ such that $2<p<q$, we assume in the following that $\pi_{i}(X)=0$ for $p \neq i<q$ and put $\pi_{p}(X)=\pi_{p}, \pi_{q}(X)=\pi_{q^{*}}$ Then $\pi_{i}\left(\Omega_{X}\right)=0$ for $p \neq i+1<q$, and $\pi_{p-1}\left(\Omega_{X}\right) \approx \pi_{p}, \pi_{q-1}\left(\Omega_{X}\right) \approx \pi_{q}$. We shall put $\pi_{p-1}\left(\Omega_{X}\right)=\pi_{p-1}, \pi_{q-1}\left(\Omega_{X}\right)=\pi_{q-1}$, and consider these groups with the canonical isomorphisms $\pi_{p} \approx \pi_{p-1}, \pi_{q} \approx \pi_{q-1}$.

Now, the spaces $X$ and $\Omega_{X}$ determine the Eilenberg-MacLane invariants $\boldsymbol{k}_{p}^{q+1}(X) \in H^{q+1}\left(\pi_{p}, p, \pi_{q}\right)$ and $\boldsymbol{k}_{p-1}^{q}\left(\Omega_{X}\right) \in H^{q}\left(\pi_{p-1}, p-1, \pi_{q-1}\right)$ respectively. As will be shown, the latter invariant $\boldsymbol{k}_{p^{-1}}^{q}\left(\Omega_{X}\right)$ is the image of the former $\boldsymbol{k}_{p}^{q+1}(X)$ under the suspension homomorphism $S$ of the cohomology groups (Theorem 2 below). Therefore, if we associate to any system $\left(\pi, \pi^{\prime}, \boldsymbol{k}_{p}^{q+1}\right)$ consisting of abelian groups $\pi, \pi^{\prime}$ and an element $\boldsymbol{k}_{p}^{q+1}$ in $H^{q+1}\left(\pi, p, \pi^{\prime}\right)$ the system $S^{*}\left(\pi, \pi^{\prime}, \boldsymbol{k}_{p}^{q \boldsymbol{t}^{1}}\right)=\left(\pi, \pi^{\prime}\right.$, $\left.S \boldsymbol{k}_{p}^{q+1}\right)$, then the correspondence $S^{*}$ has a geometrical meaning.

If $q$ is sufficiently small, we can define the inverse of this operation. When $X$ is a $C W$-complex the homotopy type of $X$ is determined by that of $\Omega_{X}{ }^{1}$ (see $\S 3$ Cor. 4 below). There is also an analogous relation about invariants of J.H. C. Whitehead which we shall show for standard complexes and standard loop spaces [5].

1) Conversely, in arcwise connected spaces, homotopy types of loop spaces are determined by those of original spaces (see $\S 5$ below). 
2. Eilenberg-MacLane complexes $K\left(\pi_{p}, p\right)$. We shall first give a short description of complexes $K\left(\pi_{p}, p\right)$ and suspension homomorphisms of Eilenberg-MacLane cohomology groups [2]. For each positive integer $n$, let $\Delta_{n}$ be a standard $n$-simplex with ordered vertices $(0, \cdots, n)$. Вy $e_{n}^{i}(i=0, \cdots, n)$ we denote the mapping of $\Delta_{n-1}$ to $\Delta_{n}$ which maps the vertices $0,1, \cdots, n-1$ of $\Delta_{n-1}$ on the vertices of $\Delta_{n}$, omitting the vertex $i$ of $\Delta_{n}$ and preserving their order. The $q$-cells of $K\left(\pi_{p}, p\right)$ are cocycles of $Z^{p}\left(\Delta_{n}, \pi_{p}\right)$. For each $g \in Z^{p}\left(\Delta_{n}, \pi_{p}\right)$ the mapping $e_{n}^{i}$ gives a cocycle $F_{i} g=g e_{n}^{i} \in Z^{p}\left(\Delta_{n-1}, \pi_{p}\right)$. We define the boundary of the $n$-cell $g$ by $\partial g=\sum_{i=0}^{n}(-1)^{i} F_{i} g$. The addition in the right hand side of the last equation is to be regarded as a formal sum of cells.

DEFinition. For each $(p-1)$-cocycle $g \in Z^{p-1}\left(\Delta_{n-1}, \pi_{p-1}\right)$ the suspended p-cocycle $\mathrm{Tg}$ is defined for each $p$-dimensional ordered simplex $\left(r_{0}, \cdots, r_{p}\right)$ of $\Delta_{n}$ by

$$
\begin{aligned}
T g\left(r_{0}, \cdots, r_{p}\right) & =g\left(r_{0}, \cdots, r_{p-1}\right) & & \text { if } r_{p}=n, \\
& =0 & & \text { if } r_{p}<n .
\end{aligned}
$$

If by $g_{0}$ we denote the cocycle which is identically zero, in the appropriate dimensions, then the suspension mapping $S$ is defined by

$$
S g=T g-g_{0} \text {. }
$$

This is a chain transformation (raising dimensions by 1) of $K\left(\pi_{p-1}, p-1\right)$ into $K\left(\pi_{p}, p\right)$ and hence induces homomorphisms

$$
S: H^{p+k}\left(\pi_{p}, p ; G\right) \rightarrow H^{p-1+k}\left(\pi_{p-1}, p-1 ; G\right)
$$

between corresponding cohomology groups, where $G$ is any abelian group and $k=0,1, \cdots$. In the following we take $\pi_{q}=\pi_{q-1}$ for $G$.

THEOREM 1. For $k<p-1$. the suspension homomorphism $S$ is an isomorphism onto. For $k=p-1$ it is an isomorphism into.

This theorem is proved by the singular cohomology theory of a fiber structure of the path space $E$, using the theory of spectral sequence (see [1], Proposition 10, p. 483). On the other hand this is shown by a purely algebraic method (see [2], [7], [8]).

3. THEOREM 2. Let $S$ be the suspension homomorphism of cohomology groups and let $\boldsymbol{k}_{p}^{q+1}(X)$ and $\boldsymbol{k}_{p-1}^{q}\left(\Omega_{X}\right)$ be Eilenberg-MacLane 
invariants of spaces $X$ and $\Omega_{X}$ respectively.

We have

$$
S\left(\boldsymbol{k}_{p}^{q+1}(X)\right)=\boldsymbol{k}_{p^{-1}}^{q}\left(\Omega_{X}\right)
$$

Proof. Let $M\left(\Omega_{X}\right)$ be a minimal complex (see [3]) in $\Omega_{X}$ based on the constant path $I \rightarrow x_{0}$. Each mapping $\sigma$ of an $(n-1)$-singular simplex $\sigma$ of $M\left(\Omega_{X}\right)$ induces a mapping $\bar{T} \sigma: \Delta_{n-1} \times I \rightarrow X$ defined as

$$
\bar{T} \sigma(x, t)=\sigma(x)(t)
$$

for any $x \in \Delta_{n-1}$ and $t \in I$. We map $\Delta_{n-1} \times I$ onto $\Delta_{n}$ by identifying the set $\Delta_{n-1} \times 1$ to the last vertex of $\Delta_{n}$. Let $i_{n}$ be this identiflcation. As $\bar{T} \sigma$ maps the set $\Delta_{n-1} \times 1$ into a point $x_{0}$, the composite mapping $\bar{T} \sigma i_{n}^{-1}$ is a continuous mapping of $\Delta_{n}$ into $X$. Therefore, it defines a singular $n$-simplex in $X$, which we denote by $\bar{S} \sigma$.

We note that a singular simplex which is a constant mapping to $x_{0}$ is called collapsed and two singular simplexes whose boundary coincide are called compatible. If for these simplexes there exists a homotopy which leaves the mapping on the boundary fixed, then we say that they are homotopic. Obviously $S$ and its inverse preserve properties stated above. Therefore, because of the definition of the minimal complex, we can take a minimal complex $M(X)$ of $X$, which contains $S\left(M\left(\Omega_{X}\right)\right.$ ) (see [3], $\left.\S 4\right)$. Let $\kappa_{X}$ be a natural simplicial transformation of $M(X)$ into $K\left(\pi_{p}, p\right)$ defined in [4] and let $\kappa_{\Omega}$ be that of $M\left(\Omega_{X}\right)$ into $K\left(\pi_{p}, p-1\right)$. Moreover, let $\bar{\kappa}_{X}$ be a simplicial transformation of the $q$-skeleton $K^{q}\left(\pi_{p}, p\right)$ of $K\left(\pi_{p}, p\right)$ into $M(X)$ which is defined by (3.1), (3.2), (3.3) of [4] and let $\bar{\kappa}_{\Omega}$ be that of $K^{q-1}\left(\pi_{p-1}, p-1\right)$ into $M\left(\Omega_{X}\right)$. Composite mappings $\kappa_{X} \bar{\kappa}_{X}$ and $\kappa_{\Omega} \bar{\kappa}_{\Omega}$ are identities. These transformations can be so constructed that commutativity relation holds in the following diagram:

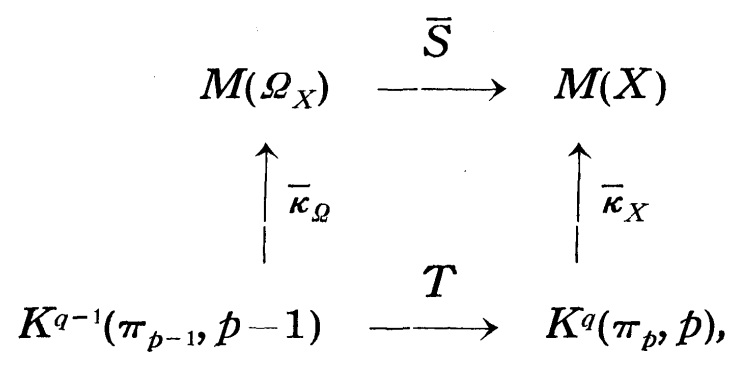


i. e. we have $\bar{S}_{\kappa_{\Omega}} g=\bar{\kappa}_{X} T g$ for any $g \in K^{q-1}\left(\pi_{p-1}, p-1\right)$. Let $\Delta_{q+1, q}$ be the $q$-skeleton of $\Delta_{q+1}$. If we attempt to continue the definition of $\bar{\kappa}_{X}$ for $(q+1)$-cell $g^{\prime}$ of $K\left(\pi_{p}, p\right)$, we can only do it for $\Delta_{q+1, q}$ so that the mapping

$$
f_{X}\left(g^{\prime}\right) ; \Delta_{q+1, q} \rightarrow X
$$

satisfies

$$
f_{X}\left(g^{\prime}\right) e_{q+1}^{i}=\bar{\kappa}_{X}\left(F_{i} g^{\prime}\right) \quad i=0, \cdots, q+1 .
$$

Since $\pi_{q}$ is not assumed to vanish, the map $f_{X}\left(g^{\prime}\right)$ in general will not be extendible to a mapping of $\Delta_{q+1}$ into $X$. Let $c f_{X}\left(g^{\prime}\right)$ be an element of $\pi_{q}$ containing $f_{X}\left(g^{\prime}\right)$. We define a cochain $k_{p}^{q+1}$ in the complex $K\left(\pi_{p}, p\right)$ by

$$
k_{p}^{q+1}\left(g^{\prime}\right)=c f_{X}\left(g^{\prime}\right) \in \pi_{q}(X) .
$$

$k_{p}^{q+1}$ is a $(q+1)$ cocycle. Its cohomology class does not depend on the choice of the simplicial transformation $\bar{\kappa}_{X}{ }^{2)}$ and it is denoted by $\boldsymbol{k}_{p}^{q+1}(X)$. Similarly a $q$-cocycle $k_{p-1}^{q}$ and its cohomology class $\boldsymbol{k}_{p-1}^{q}\left(\Omega_{X}\right)$ are defined. By $f_{\Omega}(g)$ we denote a mapping corresponding to (6) for any $q$-cell $g$ of $K\left(\pi_{p-1}, p-1\right)$. Cohomology classes $\boldsymbol{k}_{p}^{q+1}(X)$ and $\boldsymbol{k}_{p-1}^{q}\left(\Omega_{X}\right)$ do not depend on the choice of the minimal complexes (see $[4])$.

Because of the commutativity of (5), we have

$$
\begin{aligned}
f_{X}(T g) e_{i}^{q+1} & =\bar{\kappa}_{X}\left(F_{i} T g\right) \\
& =\kappa_{X}\left(T F_{i} g\right)=\bar{S}_{\kappa_{\Omega}}(g) e_{i}^{q} \quad 0 \leqq i \leqq q,
\end{aligned}
$$

and $f_{X}(S g) e_{q+1}^{q+1}$ is a constant mapping to $x_{0}$.

This yields

$$
\bar{T} f_{\Omega}(g) i_{q}^{-1}=f_{X}(T g)
$$

Therefore the element of the group $\pi_{q}(X)$ containing the mapping $f_{X}(g)$ is the image of the element of the group $\pi_{q-1}\left(\Omega_{X}\right)$ containing

2) As $\bar{\kappa}_{X}$ is determined on the $(q-1)$-skeleton $K q^{-1}\left(\pi_{p-1}, p-1\right)$ uniquely only $\bar{\kappa} g$ comes into question. 
the mapping $f_{\Omega}(g)$ under the suspension isomorphism $\Sigma$ of homotopy groups. Since groups $\pi_{q-1}\left(\Omega_{X}\right)$ and $\pi_{q}(X)$ are identified under the isomorphism in introduction, we have

$$
c f_{\Omega}(g)=c f_{X}(T g) \text {. }
$$

Using the relation $c f_{X}\left(g_{0}\right)=0$, this yields

$$
\begin{aligned}
k_{p-1}^{q}(g) & =c f_{\Omega}(g) \\
& =c f_{X}(T g) \\
& =c f_{X}(T g)-c f_{X}\left(g_{0}\right) \\
& =k_{p}^{q+1}\left(T g-g_{0}\right) \\
& =k_{p}^{q+1}(S g) \\
& =\left(S k_{p}^{q+1}\right)(g),
\end{aligned}
$$

where $S$ in the last term is a dual cochain transformation of the chain transformation $S$. Therefore

$$
\boldsymbol{k}_{p-1}^{q}\left(\Omega_{X}\right)=S\left(\boldsymbol{k}_{p}^{q+1}(X)\right)
$$

holds good. Thus the theorem is proved.

Let $K$ be a standard complex and let $\omega(K)$ be a standard loop space on the complex (see [5]). The injection mapping of the space $\omega(K)$ into the space $\Omega_{K}$ induces isomorphisms of homotopy groups of the two spaces. If we take the minimal complex $M\left(\Omega_{K}\right)$ in $\omega(K)$ particularly, we shall see that Eilenberg-MacLane invariants of two spaces are identified by the isomorphism of injection. Therefore we have the following result:

COROLLARY 3. The Eilenberg-MacLane invariant $\boldsymbol{k}_{p-1}^{q}(\omega(K))$ of the standard loop space is the image of the invariant $\boldsymbol{k}_{p}^{q+1}(K)$ of the standard complex under the suspension homomorphism $S$.

We suppose $q<2 p-1$. The Eilenberg-MacLane invariant of the space $\Omega_{X}$ determines that of the original space $X$, since the homomorphisms $S$ is one-to-one by the Theorem 1. Therefore we obtain the following result:

COROLLARY 4. Let $X, Y$ be both arcwise connected $C W$-complexes, such that $\pi_{p}(X) \approx \pi_{p}(Y), \pi_{q}(X) \approx \pi_{q}(Y)$ and $\pi_{i}(X)=\pi_{i}(Y)=0$ for $0<i<p$ 
and $p<i<q$ where $q<2 p-1$. If the spaces $\Omega_{X}, \Omega_{Y}$ have the same $q$ homotopy type, then $X, Y$ have the same $(q+1)$-type.

4. J. H. C. Whitehead invariants (see [6]).

In the following, we restrict our argument to simply connected standard complexes $K$ and their standard loop spaces $\omega(K)^{3)}$ which are also $C W$-complexes.

Let $K^{n}$ and $\omega(K)^{n}$ be $n$-skeletons of $K$ and $\omega(K)$ respectively. Then the $n$-th Whitehead invariant $\boldsymbol{l}_{n}(K)$ is defined as follows: Let $j$ be the homomorphism of $\pi_{n}\left(K^{n}\right)$ into $\pi_{n}\left(K^{n}, K^{n-1}\right)$ induced by the injection mapping and let $j^{\#}$ be its $\pi_{n}(K)$-dual. We denote by $l_{n}(K)$ the natural homomorphism

$$
\pi_{n}\left(K^{n}\right) \rightarrow \pi_{n}\left(K^{n}\right) / \partial \pi_{n+1}\left(K^{n+1}, K^{n}\right)=\pi_{n}(K),
$$

and we put

$$
\begin{gathered}
\boldsymbol{l}_{n}(K)=\left\{l_{n}(K)\right\} \\
\in \Gamma \mathrm{I}^{n}\left(K, \pi_{n}\right)=A^{\sharp}\left(\pi_{n}(K)\right) / j^{\sharp} C^{\sharp}\left(\pi_{n}(K)\right),
\end{gathered}
$$

where $A^{\sharp}\left(\pi_{n}(K)\right)$ is the group Ophom $\left(\pi_{n}\left(K^{n}\right), \pi_{n}(K)\right)^{4)}$ and $C^{\sharp}\left(\pi_{n}(K)\right)$ is the group Ophom $\left(\pi_{n}\left(K^{n}, K^{n-1}\right), \pi_{n}(K)\right)^{4}$.

Let $\alpha$ be any element of $\pi_{n}\left(\omega(K)^{n}\right)$ and let $f_{\alpha}$ be a mapping of $\left(S^{n}, s\right)$ into $\left(\omega(K)^{n}, x_{0}\right)$ contained in $\alpha$, where $S_{n}$ is an $n$-sphere, $s$ is a point of $S^{n}$, and $x_{0}$ is the base point of $\omega(K)$, i. e. the constant path $I \rightarrow x_{0}$. A suspended class $\Sigma^{\prime} \alpha$ of $\alpha$ is the class which contains the mapping $\Sigma f_{a}$ of an $(n+1)$-sphere into $K^{n+15)}$ defined as follows: we identify the subset $S^{n} \times 0 \cup S^{n} \times 1 \cup s \times I$ of $S^{n} \times I$ to a point and denote the identification mapping by $h_{n}$. We can take $h_{n}\left(S^{n} \times I\right)$ for $S^{n+1}$. The mapping $\Sigma f_{a}$ is defined by

$$
\Sigma f_{a}(y)=f_{a}(x)(t)
$$

for each point $y=h_{n}(x, t) . \quad \Sigma^{\prime \prime}$ is a homomorphism of $\pi_{n}\left(\omega(K)^{n}\right)$ into $\pi_{n+1}\left(K^{n+1}\right)$. We can extend the definition of $\Sigma^{\prime}$ to relative homotopy

3) As for singular polytopes of $M(X)$ and $M\left(\Omega_{X}\right)$, I don't know whether the results of this section hold good or not.

4) Since we assumed $\pi_{1}(K)=0$, we have Ophom $=$ Hom for the $K$.

5) This follows from the definition of the complex $\omega(K)$. 
groups similarly. It is a homomorphism of the group $\pi_{n}\left(\omega(K)^{n}\right.$, $\left.\omega(K)^{n-1}\right)$ into the group $\pi_{n+}\left(K^{n+1}, K^{n}\right)$. Then commutativity relations $\partial \Sigma^{\prime}=\Sigma \partial$ and $j \Sigma=\Sigma j$ hold good for the boundary operator $\partial$ and the injection homomorphism $j$.

The homomorphism of the group $\pi_{n}(\omega(K))$ into the group $\pi_{n+1}(K)$ induced by $\Sigma^{\prime \prime}$ coincides with the suspension isomorphism $\Sigma$ of homotopy groups. We identify these groups by the isomorphism and denote it by $\pi_{n+1}$. In the following diagramm commutativity holds good:

$$
\begin{array}{ccc}
\pi_{n}(\omega(K)) & \stackrel{\Sigma}{=} & \pi_{n+1}(K)\left(=\pi_{n+1}\right) \\
\uparrow \boldsymbol{l}_{n}(\omega(K)) & & \uparrow l_{n+1}(K) \\
\pi_{n}\left(\omega(K)^{n}\right) \stackrel{\Sigma^{\prime \prime}}{\longrightarrow} & \pi_{n+1}\left(K^{n+1}\right) .
\end{array}
$$

Let $\Sigma^{\#}$ be a $\pi_{n+1}$-dual of $\Sigma^{\prime}$ (here we do not consider operations on the groups $\pi_{n}\left(\omega(K)^{n}\right)$ and $\left.\pi_{n+1}\left(K^{n+1}\right)\right)$. Then we have

$$
\begin{aligned}
l_{n}(\omega(K)) & =l_{n+1}(K) \bigcirc \Sigma^{\prime} \\
& =\Sigma^{\#} l_{n+1}(K) .
\end{aligned}
$$

Therefore if we denote by $\Sigma^{*}$ the homomorphism of the group $\pi^{n+1}\left(\pi_{n+1}, K\right)$ into the group $\prod^{n}\left(\omega(K), \pi_{n}\right)$ induced by $\Sigma^{\#}$, then we obtain

$$
\boldsymbol{l}_{n}(\omega(K))=\Sigma^{*} \boldsymbol{l}_{n+1}(K) \text {. }
$$

This relation is analogous to (3).

5. Appendix. Finally we shall show that if two arc-wise connected, simply connected spaces are homotopy equivalent, then their loop spaces have the same property.

LEMMA 5. Let $X, Y$ be two spaces as above and let $f$ be a continuous mapping of $X$ into $Y$. $f$ induces a continuous mapping of $\Omega_{X}$ into $\Omega_{Y}$.

PROOF. Let $\tau$ be an arbitrary element of $\Omega_{X}$. We define a mapping $f_{\Omega}$ of $\Omega_{X}$ into $\Omega_{Y}$ by

$$
\left(f_{\Omega} \tau\right)(t)=f(\tau(t))
$$


for each $t \in I$. Let $C$ be a compact set of $I=[0,1]$ and let $U$ be an open set of $Y$. If by $(C, U)$ we denote an element of the open base of $\Omega_{Y}$, determined by $C$ and $U$, then $\left(C, f^{-1}(U)\right)$ is an open set of $\Omega_{X}$. Since we have $f_{\Omega}^{-1}(C, U)=\left(C, f^{-1}(U)\right), f_{\Omega}$ is continuous.

LemMA 6. Let $F: X \times I \rightarrow Y$ be a homotopy. This mapping induces a homotopy of mappings of $\Omega_{X}$ into $\Omega_{Y}$ defined by the above lemma.

PROOF. Let $\tau$ be an arbitrary element of $\Omega_{X}$. We define a mapping $F_{\Omega}$ of $\Omega_{X} \times I$ into $\Omega_{Y}$ by

$$
F_{Q}(\tau, s)(t)=F(\tau(t), s)
$$

for any $t$ and $s$ in $I$. Let $(C, U)$ be an arbitrary element of the open base in $\Omega_{Y}$ which contains $F(\tau, s)$. An inverse image $F^{-1}(U)$ of $U$ is an open set in $X \times I$ and it contains $(\tau(C), s) . \quad(\tau(C), s)$ is a compact set, therefore there exists a finite open covering $\left(U_{i},\left(s-\varepsilon_{i}, s+\varepsilon_{i}\right)\right)$ contained in $F^{-1}(U)$ for each $i$, where $\varepsilon_{i}$ is a positive real number. We put Min $\varepsilon_{i}=\varepsilon>0$ and $V=\left(\left(C, \cup_{i} U_{i}\right),(s-\varepsilon, s+\varepsilon)\right)$. We have $(\tau, s) \in V$ and $F_{\Omega}(V) \subset(C, U)$. Therefore $F_{\Omega}$ is continuous.

THEOREM 7. If two spaces $X$ and $Y$ are homotopy equivalent then their loop spaces have the same property.

This is an immediate consequence of the two lemmas stated above.

\section{Mathematical Institute, Tohoku University.}

\section{References}

1. J.-P. Serre, Homologie singulière des espace fibrés et Applications, Ann. of Math., 54 (1951), pp. 425-505.

2. S. Eilenberg and S. MacLane, Cohomology theory of abelian groups and homotopy theory I-III, Proc. N. A. S., U. S. A., 35 (1950), pp. 443-447 and 657-663, 37 (1951), pp. 307-310.

3. S. Eilenberg and J. A. Zilber, Semi-simplicial complexes and singular homology, Ann. of Math., 51 (1950), pp. 499-513.

4. S. Eilenberg and S. MacLane, Relations between homology and homotopy groups of spaces II, Ann. of Math., 51 (1950), pp. 514-533.

5. H. Toda, Topology of standard path spaces and homotopy theory I, Proc. of Japan Acad., 29, pp. 229-304. 
6. J. H.C. Whitehead, The G-dual of semi-exact couple, Proc. of the London Math. Soc., 3rd Ser. vol. III (1953), 385-416.

7. S. Eilenberg and S. MacLane, On the groups $H(\pi, n)$ I, Ann. of Math., 58 (1953), pp. 55-166.

8. H. Cartan, Sur les groupes d'Eilenberg-MacLane $H(\pi, n)$ I, Methode des construction, Proc. N. A.S., U.S. A., 40 (1954), pp. 467-471. 